\title{
Supporting Information for Near-field Terahertz Sensing of Hela cells and \\ Pseudomonas Based on Monolithic Integrated Metamaterials with Spintronic Terahertz Emitter
}

Zhongyang Bai, ${ }^{\gamma a, b}$ Yongshan Liu, ${ }^{\gamma a, b}$ Ruru Kong, ${ }^{a, c}$ Tianxiao Nie, ${ }^{* a, b}$ Yun Sun, ${ }^{a, c}$ Helin Li, ${ }^{a, b}$ Tong Sun, ${ }^{a, b}$ Chandan Pandey, ${ }^{a, b}$ Yining Wang, ${ }^{a}$ Haoyi Zhang, ${ }^{a}$ Qinglin Song, ${ }^{b}$ Guozhen Liu, ${ }^{e}$ Michael Kraft, ${ }^{f}$ Weisheng Zhao, ${ }^{a, b}$ Xiaojun $W u,{ }^{* d, g}$ Lianggong Wen, ${ }^{* a, b}$

a School of Microelectronics, Beihang University, Beijing, 100191, China.

b Beihang-Goertek Joint Microelectronics Institute, Qingdao Research Institute of Beihang University, Qingdao, 266000, China.

c Hefei Innovation Research Institute, Beihang University, Hefei, 230013, China.

d School of Electronics and Information Engineering, Beihang University, Beijing, 100191, China.

e Graduate School of Biomedical Engineering, ARC Centre of Excellence in Nanoscale BioPhotonics (CNBP), Faculty of Engineering, University of New South Wales, Sydney, NSW, 2052, Australia.

f ESAT-MICAS, KU Leuven, Kasteelpark Arenberg 10, 3001 Leuven, Belgium

g Huazhong University of Science and Technology, Wuhan National Laboratory for Optoelectronics, Wuhan 430074, China.

* Corresponding author:

Lianggong Wen Email: wenlg@bhqditi.com

Xiaojun Wu_Email: xiaojunwu@buaa.edu.cn

Tianxiao Nie Email: nietianxiao@buaa.edu.cn 
${ }^{\dagger}$ These authors contributed equally to this paper.

Table S1 summerizes the sensitivity performance comparison between the fully integrated TEB (terahertz emission biosensor) and other purposed biosensors. The sensitivity defined as

$$
\text { Sensitivity }=\frac{\Delta f}{f_{0}} \times 100
$$

$f_{0}$ represents the initial resonance frequency of TEB, while $\Delta f$ represents the frequency shift of the resonance frequency after adding samples of different concentrations on the surface of TEB. This shows the intergrated TEB exhibits a excellent sensitivity of 15.68 , compared to other biosensor.

Table S1.Comparison of structural and sensitivity performance of other purposed biosensors and this work

\begin{tabular}{|c|c|c|c|c|}
\hline Reference & Resonator topology & $\begin{array}{l}\text { Resonance } \\
\text { frequency }\end{array}$ & $\begin{array}{c}\text { Frequency } \\
\text { shift }\end{array}$ & Sensitivity \\
\hline Ding et al. (2015) & Asymmetric four Split ring & $1.24 \mathrm{THz}$ & $60 \mathrm{GHz}$ & 4.84 \\
\hline Chen et al. (2016) & $\begin{array}{c}\text { Symmetric Double Split } \\
\text { rings }\end{array}$ & $0.83 \mathrm{THz}$ & $63 \mathrm{GHz}$ & 7.59 \\
\hline Chen et al. (2017) & $\begin{array}{c}\text { Asymmetric dual wire } \\
\text { resonator }\end{array}$ & $1.73 \mathrm{THz}$ & $\sim 220 \mathrm{GHz}$ & 12.71 \\
\hline Yan et al. (2019) & $\begin{array}{l}\text { Double splits ring } \\
\text { resonators (DSRR) }\end{array}$ & $1.65 \mathrm{THz}$ & $90 \mathrm{GHz}$ & 5.45 \\
\hline This Work & $\begin{array}{c}\text { Double splits ring } \\
\text { resonators with } \\
\text { spintornic terahertz } \\
\text { emitter }\end{array}$ & $1.02 \mathrm{THz}$ & $\sim 160 \mathrm{GHz}$ & 15.68 \\
\hline
\end{tabular}

